 \\ TATRA \\ MOUNTaiNS \\ Mathematical Publications}

DOI: $10.2478 / \mathrm{tmmp}-2014-0021$

Tatra Mt. Math. Publ. 59 (2014), 93-103

\title{
SUBGROUPS OF FINITE ABELIAN GROUPS HAVING RANK TWO VIA GOURSAT'S LEMMA
}

\author{
LÁSZLÓ TÓTH
}

\begin{abstract}
Using Goursat's lemma for groups, a simple representation and the invariant factor decompositions of the subgroups of the group $\mathbb{Z}_{m} \times \mathbb{Z}_{n}$ are deduced, where $m$ and $n$ are arbitrary positive integers. As consequences, explicit formulas for the total number of subgroups, the number of subgroups with a given invariant factor decomposition, and the number of subgroups of a given order are obtained.
\end{abstract}

\section{Introduction}

Let $\mathbb{Z}_{m}$ denote the additive group of residue classes modulo $m$ and consider the direct product $\mathbb{Z}_{m} \times \mathbb{Z}_{n}$, where $m, n \in \mathbb{N}:=\{1,2, \ldots\}$ are arbitrary. Note that this group is isomorphic to $\mathbb{Z}_{\operatorname{gcd}(m, n)} \times \mathbb{Z}_{\mathrm{lcm}(m, n)}$. If $\operatorname{gcd}(m, n)=1$, then it is cyclic, isomorphic to $\mathbb{Z}_{m n}$. If $\operatorname{gcd}(m, n)>1$, then $\mathbb{Z}_{m} \times \mathbb{Z}_{n}$ has rank two. We recall that a finite abelian group of order $>1$ has rank $r$ if it is isomorphic to $\mathbb{Z}_{n_{1}} \times \cdots \times \mathbb{Z}_{n_{r}}$, where $n_{1}, \ldots, n_{r} \in \mathbb{N} \backslash\{1\}$ and $n_{j} \mid n_{j+1}(1 \leq j \leq r-1)$, which is the invariant factor decomposition of the given group. Here the number $r$ is uniquely determined and represents the minimal number of generators of the group. For general accounts on finite abelian groups see, e.g., [10], 14].

In this paper we apply Goursat's lemma for groups, see Section 2, to derive a simple representation and the invariant factor decompositions of the subgroups of $\mathbb{Z}_{m} \times \mathbb{Z}_{n}$ (Theorem 3.1). These are new results, as far as we know. Then, we deduce as consequences, by purely number theoretical arguments, explicit formulas for the total number of subgroups of $\mathbb{Z}_{m} \times \mathbb{Z}_{n}$ (Theorem 4.1), the number of its subgroups of a given order (Theorem 4.3) and the number of subgroups with a given invariant factor decomposition (Theorem 4.5, which is another new result). The number of cyclic subgroups (of a given order) is

(C) 2014 Mathematical Institute, Slovak Academy of Sciences.

2010 Mathematics Subject Classification: 20K01, 20K27, 11A25.

Keyw ords: cyclic group, direct product, subgroup, number of subgroups, Goursat's lemma, finite abelian group of rank two. 


\section{LÁSZLÓ TÓTH}

also treated (Theorems 4.6 and 4.7). Furthermore, in Section 5 a table for the subgroups of the group $\mathbb{Z}_{12} \times \mathbb{Z}_{18}$ is given to illustrate the applicability of our identities.

The results of Theorems 4.1 and 4.3 generalize and put in more compact forms those of G. Călugăr e anu [4, J. Petrillo [13] and M. Tărnăuceanu [15], obtained for $p$-groups of rank two, and included in Corollaries 4.2 and 4.4. We remark that both the papers [4] and [13] applied Goursat's lemma for groups (the first one in a slightly different form), while the paper [15] used a different approach based on properties of certain attached matrices.

Another representation of the subgroups of $\mathbb{Z}_{m} \times \mathbb{Z}_{n}$, and the formulas of Theorems 4.1, 4.3 and 4.6, but not Theorem 4.5, were also derived in [7] using different group theoretical arguments. That representation and the formula of Theorem 4.1 was generalized to the case of the subgroups of the group $\mathbb{Z}_{m} \times \mathbb{Z}_{n} \times \mathbb{Z}_{r}(m, n, r \in \mathbb{N})$ [], using similar arguments, which are different from those of the present paper.

Note that in the case $m=n$ the subgroups of $\mathbb{Z}_{n} \times \mathbb{Z}_{n}$ play an important role in the field of applied time-frequency analysis (cf. [7]). See [1] for asymptotic results on the number of subgroups of $\mathbb{Z}_{m} \times \mathbb{Z}_{n}$.

Throughout the paper we use the following standard notations: $\tau(n)$ is the number of the positive divisors of $n$, $\phi$ denotes Euler's totient function, $\mu$ is the Möbius function, $*$ is the Dirichlet convolution of arithmetic functions.

\section{Goursat's lemma for groups}

Goursat's lemma for groups [6, p. 43-48] can be stated as follows:

Proposition 2.1. Let $G$ and $H$ be arbitrary groups. Then there is a bijection between the set $S$ of all subgroups of $G \times H$ and the set $T$ of all 5 -tuples $(A, B, C, D, \Psi)$, where $B \unlhd A \leq G, D \unlhd C \leq H$ and $\Psi: A / B \rightarrow C / D$ is an isomorphism (here $\leq$ denotes subgroup and $\unlhd$ denotes normal subgroup). More precisely, the subgroup corresponding to $(A, B, C, D, \Psi)$ is

$$
K=\{(g, h) \in A \times C: \Psi(g B)=h D\} .
$$

Corollary 2.2. Assume that $G$ and $H$ are finite groups and that the subgroup $K$ of $G \times H$ corresponds to the 5 -tuple $\left(A_{K}, B_{K}, C_{K}, D_{K}, \Psi_{K}\right)$ under this bijection. Then one has $\left|A_{K}\right| \cdot\left|D_{K}\right|=|K|=\left|B_{K}\right| \cdot\left|C_{K}\right|$.

For the history, proof, discussion, applications and a generalization of Goursat's lemma see [1], 2], 5], [9, [12], [13. Corollary 2.2 is given in [5, Cor. 3]. 


\section{Representation of the subgroups of $\mathbb{Z}_{m} \times \mathbb{Z}_{n}$}

For every $m, n \in \mathbb{N}$ let

$J_{m, n}:=\left\{(a, b, c, d, \ell) \in \mathbb{N}^{5}: a|m, b| a, c|n, d| c, \frac{a}{b}=\frac{c}{d}, \ell \leq \frac{a}{b}, \operatorname{gcd}\left(\ell, \frac{a}{b}\right)=1\right\}$.

Using the condition

we deduce

$$
a / b=c / d
$$

$$
\operatorname{lcm}(a, c)=\operatorname{lcm}(a, a d / b)=\operatorname{lcm}(a d / d, a d / b)=a d / \operatorname{gcd}(b, d) .
$$

That is, $\operatorname{gcd}(b, d) \cdot \operatorname{lcm}(a, c)=a d$. Also, $\operatorname{gcd}(b, d) \mid \operatorname{lcm}(a, c)$.

For $(a, b, c, d, \ell) \in J_{m, n}$ define

$$
K_{a, b, c, d, \ell}:=\left\{\left(i \frac{m}{a}, i \ell \frac{n}{c}+j \frac{n}{d}\right): 0 \leq i \leq a-1,0 \leq j \leq d-1\right\} .
$$

TheOREM 3.1. Let $m, n \in \mathbb{N}$.

i) The map $(a, b, c, d, \ell) \mapsto K_{a, b, c, d, \ell}$ is a bijection between the set $J_{m, n}$ and the set of subgroups of $\left(\mathbb{Z}_{m} \times \mathbb{Z}_{n},+\right)$.

ii) The invariant factor decomposition of the subgroup $K_{a, b, c, d, \ell}$ is

$$
K_{a, b, c, d, \ell} \simeq \mathbb{Z}_{\operatorname{gcd}(b, d)} \times \mathbb{Z}_{\operatorname{lcm}(a, c)} .
$$

iii) The order of the subgroup $K_{a, b, c, d, \ell}$ is ad and its exponent is $\operatorname{lcm}(a, c)$.

iv) The subgroup $K_{a, b, c, d, \ell}$ is cyclic if and only if $\operatorname{gcd}(b, d)=1$.

Figure 1 represents the subgroup $K_{6,2,18,6,1}$ of $\mathbb{Z}_{12} \times \mathbb{Z}_{18}$. It has order 36 and is isomorphic to $\mathbb{Z}_{2} \times \mathbb{Z}_{18}$.

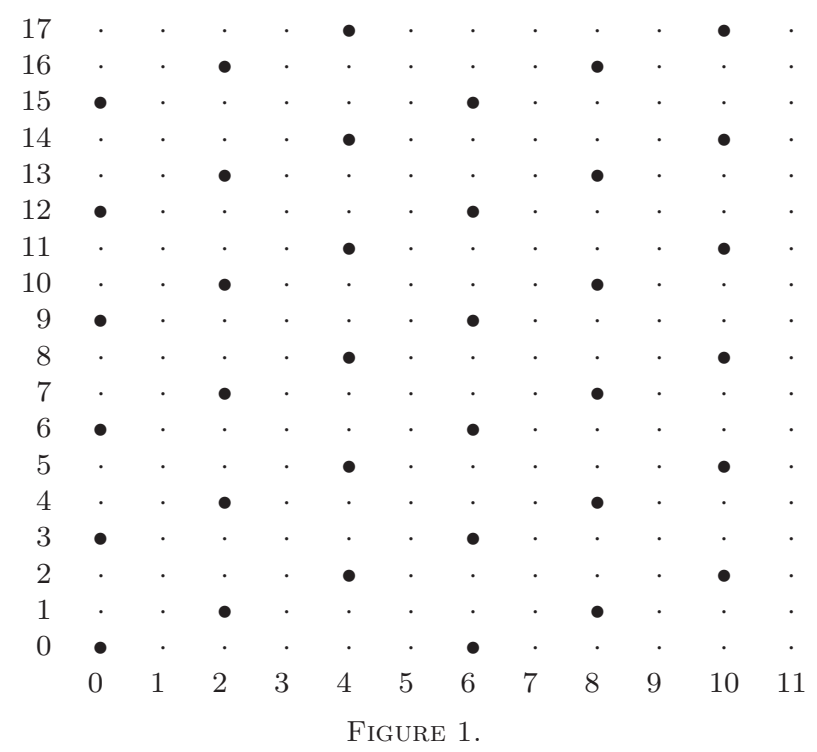




\section{LÁSZLÓ TÓTH}

Proof. i) Apply Goursat's lemma for the groups $G=\mathbb{Z}_{m}$ and $H=\mathbb{Z}_{n}$. We only need the following simple additional properties:

- all subgroups and all quotient groups of $\mathbb{Z}_{n}(n \in \mathbb{N})$ are cyclic;

- for every $n \in \mathbb{N}$ and every $a \mid n, a \in \mathbb{N}$, there is precisely one (cyclic) subgroup of order $a$ of $\mathbb{Z}_{n}$;

- the number of automorphisms of $\mathbb{Z}_{n}$ is $\phi(n)$ and they can be represented as $f: \mathbb{Z}_{n} \rightarrow \mathbb{Z}_{n}, f(x)=\ell x$, where $1 \leq \ell \leq n, \operatorname{gcd}(\ell, n)=1$.

With the notations of Proposition 2.1, let $|A|=a,|B|=b,|C|=c,|D|=d$, where $a|m, b| a, c|n, d| c$. Writing explicitly the corresponding subgroups and quotient groups we deduce:

$$
\begin{aligned}
A & =\langle m / a\rangle=\left\{0, \frac{m}{a}, 2 \frac{m}{a}, \ldots,(a-1) \frac{m}{a}\right\} \leq \mathbb{Z}_{m}, \\
B & =\langle m / b\rangle=\left\{0, \frac{m}{b}, 2 \frac{m}{b}, \ldots,(b-1) \frac{m}{b}\right\} \leq A, \\
A / B & =\left\langle\frac{m}{a}+B\right\rangle=\left\{B, \frac{m}{a}+B, 2 \frac{m}{a}+B, \ldots,\left(\frac{a}{b}-1\right) \frac{m}{a}+B\right\},
\end{aligned}
$$

and similarly

$$
\begin{aligned}
C & =\langle n / c\rangle=\left\{0, \frac{n}{c}, 2 \frac{n}{c}, \ldots,(c-1) \frac{n}{c}\right\} \leq \mathbb{Z}_{n}, \\
D & =\langle n / d\rangle=\left\{0, \frac{n}{d}, 2 \frac{n}{d}, \ldots,(d-1) \frac{n}{d}\right\} \leq C, \\
C / D & =\left\langle\frac{n}{c}+D\right\rangle=\left\{D, \frac{n}{c}+D, 2 \frac{n}{c}+D, \ldots,\left(\frac{c}{d}-1\right) \frac{n}{c}+D\right\} .
\end{aligned}
$$

Now, in the case $a / b=c / d$ the values of the isomorphisms $\Psi: A / B \rightarrow C / D$ are

$$
\Psi\left(i \frac{m}{a}+B\right)=i \ell \frac{n}{c}+D, \quad 0 \leq i \leq \frac{a}{b}-1,
$$

where $1 \leq \ell \leq a / b, \operatorname{gcd}(\ell, a / b)=1$. Using (11) we deduce that the corresponding subgroup is

$$
\begin{aligned}
K & =\left\{\left(i \frac{m}{a}, k \frac{n}{c}\right) \in A \times C: \Psi\left(i \frac{m}{a}+B\right)=k \frac{n}{c}+D\right\} \\
& =\left\{\left(i \frac{m}{a}, k \frac{n}{c}\right): 0 \leq i \leq a-1,0 \leq k \leq c-1, i \ell \frac{n}{c}+D=k \frac{n}{c}+D\right\},
\end{aligned}
$$

where the last condition is equivalent, in turn, to $k n / c \equiv i \ell n / c(\bmod n / d)$, $k \equiv i \ell(\bmod c / d)$, and finally $k=i \ell+j c / d, 0 \leq j \leq d-1$. Hence,

$$
K=\left\{\left(i \frac{m}{a},\left(i \ell+j \frac{c}{d}\right) \frac{n}{c}\right): 0 \leq i \leq a-1,0 \leq j \leq d-1\right\},
$$

and the proof of the representation formula is complete. 
ii-iii) It is clear from (3) that $\left|K_{a, b, c, d, \ell}\right|=a d=b c$ (or cf. Corollary 2.2). Next we deduce the exponent of $K_{a, b, c, d, \ell}$. According to (3) the subgroup $K_{a, b, c, d, \ell}$ is generated by the elements $(0, n / d)$ and $(m / a, \ell n / c)$. Here the order of $(0, n / d)$ is $d$. To obtain the order of $(m / a, \ell n / c)$ note the following properties:

(1) $m \mid r(m / a)$ if and only if $m / \operatorname{gcd}(m, m / a) \mid r$ if and only if $a \mid r$, and the least such $r \in \mathbb{N}$ is $a$,

(2) $n \mid t(\ell n / c)$ if and only if $n / \operatorname{gcd}(n, \ell n / c) \mid t$ if and only if $c / \operatorname{gcd}(\ell, c) \mid t$, and the least such $t \in \mathbb{N}$ is $c / \operatorname{gcd}(\ell, c)$.

Therefore the order of $(m / a, \ell n / c)$ is $\operatorname{lcm}(a, c / \operatorname{gcd}(\ell, c))$. We deduce that the exponent of $K_{a, b, c, d, \ell}$ is

$$
\begin{aligned}
& \operatorname{lcm}\left(d, \operatorname{lcm}\left(a, \frac{c}{\operatorname{gcd}(\ell, c)}\right)\right)=\operatorname{lcm}\left(d, a, \frac{c}{\operatorname{gcd}(\ell, c)}\right) \\
& =\operatorname{lcm}\left(\frac{a c}{a c / d}, \frac{a c}{c}, \frac{a c}{a \operatorname{gcd}(\ell, c)}\right)=\frac{a c}{\operatorname{gcd}(a c / d, c, a \operatorname{gcd}(\ell, c))} \\
& =\frac{a c}{\operatorname{gcd}(c, a \operatorname{gcd}(c / d, \operatorname{gcd}(\ell, c)))}=\frac{a c}{\operatorname{gcd}(c, a \operatorname{gcd}(\ell, \operatorname{gcd}(c / d, c)))} \\
& =\frac{a c}{\operatorname{gcd}(c, a \operatorname{gcd}(\ell, c / d))}=\frac{a c}{\operatorname{gcd}(a, c)}=\operatorname{lcm}(a, c),
\end{aligned}
$$

using that $\operatorname{gcd}(\ell, c / d)=1$, cf. (2) .

Now $K_{a, b, c, d, \ell}$ is a subgroup of the abelian group $\mathbb{Z}_{m} \times \mathbb{Z}_{n}$ having rank $\leq 2$. Therefore, $K_{a, b, c, d, \ell}$ has also rank $\leq 2$. That is,

$$
K_{a, b, c, d, \ell} \simeq \mathbb{Z}_{u} \times \mathbb{Z}_{v}
$$

for unique $u$ and $v$, where $u \mid v$ and $u v=a d$. Hence the exponent of $K_{a, b, c, d, \ell}$ is $\operatorname{lcm}(u, v)=v$. We obtain

This gives (41).

$$
v=\operatorname{lcm}(a, c) \quad \text { and } \quad u=a d / \operatorname{lcm}(a, c)=\operatorname{gcd}(b, d) .
$$

iv) Clear from ii). This follows also from a general result given in [2, Th. 4.2].

Remark 3.2. One can also write $K_{a, b, c, d, \ell}$ in the form

$$
\left\{\left(i \frac{m}{a}, i \ell \frac{n}{c}+j \frac{n}{d}\right): 0 \leq i \leq a-1, j_{i} \leq j \leq j_{i}+d-1\right\},
$$

where $j_{i}=-\lfloor i \ell d / c\rfloor$. Then for the second coordinate one has

for every given $i$ and $j$.

$$
0 \leq i \ell \frac{n}{c}+j \frac{n}{d} \leq n-1
$$




\section{LÁSZLÓ TÓTH}

\section{Number of subgroups}

According to Theorem 3.1 the total number $s(m, n)$ of subgroups of $\mathbb{Z}_{m} \times \mathbb{Z}_{n}$ can be obtained by counting the elements of the set $J_{m, n}$, which is now a purely number theoretical question.

TheOREM 4.1. For every $m, n \in \mathbb{N}, s(m, n)$ is given by

$$
\begin{aligned}
s(m, n) & =\sum_{i|m, j| n} \operatorname{gcd}(i, j) \\
& =\sum_{t \mid \operatorname{gcd}(m, n)} \phi(t) \tau\left(\frac{m}{t}\right) \tau\left(\frac{n}{t}\right) .
\end{aligned}
$$

P r o of. We have

$$
s(m, n)=\left|J_{m, n}\right|=\sum_{\substack{a|m \\ b| a}} \sum_{\substack{c|n \\ d| c}} \sum_{a / b=c / d=e} \phi(e) .
$$

Let $m=a x, a=b y, n=c z, c=d t$. Then, by the condition $a / b=c / d=e$ we have $y=t=e$. Rearranging the terms of (7),

$$
\begin{aligned}
& s(m, n)=\sum_{b x e=m} \sum_{d z e=n} \phi(e)=\sum_{\substack{i x=m \\
j z=n}} \sum_{\substack{b e=i \\
d e=j}} \phi(e) \\
& =\sum_{\substack{i|m \\
j| n}} \sum_{e \mid \operatorname{gcd}(i, j)} \phi(e)=\sum_{\substack{i|m \\
j| n}} \operatorname{gcd}(i, j),
\end{aligned}
$$

finishing the proof of (5). To obtain the formula (6) write (8) as follows:

$$
\begin{aligned}
& s(m, n)=\sum_{\substack{e k=m \\
e \ell=n}} \phi(e) \sum_{\begin{array}{c}
b x=k \\
d z=\ell
\end{array}} 1=\sum_{\begin{array}{c}
e k=m \\
e \ell=n
\end{array}} \phi(e) \tau(k) \tau(\ell) \\
& =\sum_{e \mid \operatorname{gcd}(m, n)} \phi(e) \tau\left(\frac{m}{e}\right) \tau\left(\frac{n}{e}\right) .
\end{aligned}
$$

Note that (5) is a special case of an identity deduced in [3] by different arguments.

Corollary 4.2 (4, [13, Prop. 2], 15, Th. 3.3]). The total number of subgroups of the p-group $\mathbb{Z}_{p^{a}} \times \mathbb{Z}_{p^{b}}(1 \leq a \leq b)$ of rank two is given by

$$
s\left(p^{a}, p^{b}\right)=\frac{(b-a+1) p^{a+2}-(b-a-1) p^{a+1}-(a+b+3) p+(a+b+1)}{(p-1)^{2}} .
$$

Now consider $s_{\delta}(m, n)$, denoting the number of subgroups of order $\delta$ of $\mathbb{Z}_{m} \times \mathbb{Z}_{n}$. 
TheOREM 4.3. For every $m, n, \delta \in \mathbb{N}$ such that $\delta \mid m n$,

$$
s_{\delta}(m, n)=\sum_{\substack{i|\operatorname{gcd}(m, \delta) \\ j| \operatorname{gcd}(n, \delta) \\ \delta \mid i j}} \phi\left(\frac{i j}{\delta}\right) .
$$

P r o of. Similar to the above proof. We have

$$
s_{\delta}(m, n)=\sum_{\substack{a|m \\
b| a}} \sum_{\substack{c|n \\
d| c}} \sum_{\begin{array}{c}
a / b=c / d=e \\
a d=b c=\delta
\end{array}} \phi(e)=\sum_{b x e=m} \sum_{d z e=n} \sum_{b d e=\delta} \phi(e)=\sum_{\substack{i x=m \\
j z=n}} \sum_{\substack{b e=i \\
d e=j \\
b d e=\delta}} \phi(e),
$$

where the only term of the inner sum is obtained for $e=i j / \delta$ provided that $\delta|i j, i| \delta$ and $j \mid \delta$. This gives (10).

CoRollaRY 4.4 ([15, Th. 3.3]). The number of subgroups of order $p^{c}$ of the p-group $\mathbb{Z}_{p^{a}} \times \mathbb{Z}_{p^{b}}(1 \leq a \leq b)$ is given by

$$
s_{p^{c}}\left(p^{a}, p^{b}\right)= \begin{cases}\frac{p^{c+1}-1}{p-1}, & c \leq a \leq b, \\ \frac{p^{a+1}-1}{p-1}, & a \leq c \leq b, \\ \frac{p^{a+b-c+1}-1}{p-1}, & a \leq b \leq c \leq a+b .\end{cases}
$$

The number of subgroups of $\mathbb{Z}_{m} \times \mathbb{Z}_{n}$ with a given isomorphism type $\mathbb{Z}_{A} \times \mathbb{Z}_{B}$ is given by the following formula.

Theorem 4.5. Let $m, n \in \mathbb{N}$ and let $A, B \in \mathbb{N}$ such that $A|B, A B| m n$. Let $A \mid \operatorname{gcd}(m, n)$, Then the number $N_{A, B}(m, n)$ of subgroups of $\mathbb{Z}_{m} \times \mathbb{Z}_{n}$, which are isomorphic to $\mathbb{Z}_{A} \times \mathbb{Z}_{B}$ is given by

$$
N_{A, B}(m, n)=\sum_{\substack{i|m, j| n \\ A B \mid i j \\ \operatorname{lcm}(i, j)=B}} \phi\left(\frac{i j}{A B}\right) .
$$

If $A \nmid \operatorname{gcd}(m, n)$, then $N_{A, B}(m, n)=0$.

P r o of. Using Proposition 3.1/ ii) we have

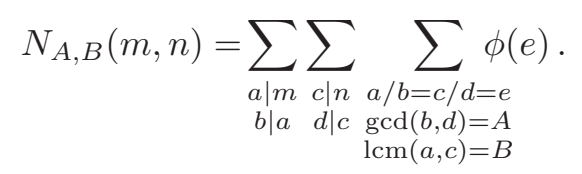

Here the condition $\operatorname{gcd}(b, d)=A$ implies that $A \mid m$ and $A \mid n$. In this case

$$
N_{A, B}(m, n)=\sum_{\substack{b x e=m \\
d z e=n}} \sum_{\begin{array}{c}
\operatorname{gcd}(b, d)=A \\
b d e=A B
\end{array}} \phi(e)=\sum_{\substack{i x=m \\
j z=n}} \sum_{\begin{array}{c}
b e=i \\
d e=j \operatorname{gcd}(b, d)=A
\end{array}} \phi(e),
$$




\section{LÁSZLÓ TÓTH}

where the only term of the inner sum is obtained for $e=i j /(A B)$ provided that $A B|i j, i| A B$ and $j \mid A B$.

Remark: It is also possible that $N_{A, B}(m, n)=0$ even when all the conditions in the hypotheses are satisfied, e.g., see the Table in Section 5.

From the representation of the cyclic subgroups given in Proposition 3.1/ iv) we also deduce the next result.

TheOREM 4.6. For every $m, n \in \mathbb{N}$ the number $c(m, n)$ of cyclic subgroups of $\mathbb{Z}_{m} \times \mathbb{Z}_{n}$ is

$$
\begin{aligned}
c(m, n) & =\sum_{i|m, j| n} \phi(\operatorname{gcd}(i, j)) \\
& =\sum_{t \mid \operatorname{gcd}(m, n)}(\mu * \phi)(t) \tau\left(\frac{m}{t}\right) \tau\left(\frac{n}{t}\right) .
\end{aligned}
$$

P r o of. Similar to the above proofs, using that for the cyclic subgroups one has $\operatorname{gcd}(b, d)=1$.

Let $c_{\delta}(m, n)$ denote the number of cyclic subgroups of order $\delta$ of $\mathbb{Z}_{m} \times \mathbb{Z}_{n}$.

TheOREM 4.7. For every $m, n, \delta \in \mathbb{N}$ such that $\delta \mid m n$,

$$
c_{\delta}(m, n)=\sum_{\substack{i|m, j| n \\ \operatorname{lcm}(i, j)=\delta}} \phi(\operatorname{gcd}(i, j)) .
$$

Pr o of. This is a direct consequence of (12) obtained in the cases $A=1$ and $B=\delta$.

In the paper [7] the identities (5), (6), (10), (13) and (14) were derived using another approach. The identity (13), as a special case of a formula valid for arbitrary finite abelian groups, was obtained by the author [16], 17] using different arguments. Finally, we remark that the functions $(m, n) \mapsto s(m, n)$ and $(m, n) \mapsto c(m, n)$ are multiplicative, viewed as arithmetic functions of two variables. See [7], [18] for details.

\section{Table of the subgroups of $\mathbb{Z}_{12} \times \mathbb{Z}_{18}$}

To illustrate our results we describe the subgroups of the group $\mathbb{Z}_{12} \times \mathbb{Z}_{18}$ ( $m=12, n=18)$. According to Proposition 4.5, there exist subgroups isomorphic to $\mathbb{Z}_{A} \times \mathbb{Z}_{B}(A \mid B)$ only if $A \mid \operatorname{gcd}(12,18)=6$, that is

$$
A \in\{1,2,3,6\} \text { and } A B \mid 12 \cdot 18=216 .
$$


TABLE 1. Table of the subgroups of $\mathbb{Z}_{12} \times \mathbb{Z}_{18}$.

\begin{tabular}{|l|r||l|r|}
\hline Total number subgroups & 80 & & \\
\hline Number subgroups order 1 & 1 & Number subgroups order 18 & 12 \\
\hline Number subgroups order 2 & 3 & Number subgroups order 24 & 4 \\
\hline Number subgroups order 3 & 4 & Number subgroups order 27 & 1 \\
\hline Number subgroups order 4 & 3 & Number subgroups order 36 & 12 \\
\hline Number subgroups order 6 & 12 & Number subgroups order 54 & 3 \\
\hline Number subgroups order 8 & 1 & Number subgroups order 72 & 4 \\
\hline Number subgroups order 9 & 4 & Number subgroups order 108 & 3 \\
\hline Number subgroups order 12 & 12 & Number subgroups order 216 & 1 \\
\hline Number cyclic subgroups & 48 & Number noncyclic subgroups & 32 \\
\hline Number subgroups $\simeq \mathbb{Z}_{1}$ & 1 & Number subgroups $\simeq \mathbb{Z}_{2} \times \mathbb{Z}_{2}$ & 1 \\
\hline Number subgroups $\simeq \mathbb{Z}_{2}$ & 3 & Number subgroups $\simeq \mathbb{Z}_{2} \times \mathbb{Z}_{4}$ & 1 \\
\hline Number subgroups $\simeq \mathbb{Z}_{3}$ & 4 & Number subgroups $\simeq \mathbb{Z}_{2} \times \mathbb{Z}_{6}$ & 4 \\
\hline Number subgroups $\simeq \mathbb{Z}_{4}$ & 2 & Number subgroups $\simeq \mathbb{Z}_{2} \times \mathbb{Z}_{12}$ & 4 \\
\hline Number subgroups $\simeq \mathbb{Z}_{6}$ & 12 & Number subgroups $\simeq \mathbb{Z}_{2} \times \mathbb{Z}_{18}$ & 3 \\
\hline Number subgroups $\simeq \mathbb{Z}_{9}$ & 3 & Number subgroups $\simeq \mathbb{Z}_{2} \times \mathbb{Z}_{36}$ & 3 \\
\hline Number subgroups $\simeq \mathbb{Z}_{12}$ & 8 & Number subgroups $\simeq \mathbb{Z}_{3} \times \mathbb{Z}_{3}$ & 1 \\
\hline Number subgroups $\simeq \mathbb{Z}_{18}$ & 9 & Number subgroups $\simeq \mathbb{Z}_{3} \times \mathbb{Z}_{6}$ & 3 \\
\hline Number subgroups $\simeq \mathbb{Z}_{36}$ & 6 & Number subgroups $\simeq \mathbb{Z}_{3} \times \mathbb{Z}_{9}$ & 1 \\
\hline & & Number subgroups $\simeq \mathbb{Z}_{3} \times \mathbb{Z}_{12}$ & 2 \\
\hline & & Number subgroups $\simeq \mathbb{Z}_{3} \times \mathbb{Z}_{18}$ & 3 \\
\hline & & Number subgroups $\simeq \mathbb{Z}_{3} \times \mathbb{Z}_{36}$ & 2 \\
\hline & & Number subgroups $\simeq \mathbb{Z}_{6} \times \mathbb{Z}_{6}$ & 1 \\
\hline & Number subgroups $\simeq \mathbb{Z}_{6} \times \mathbb{Z}_{12}$ & 1 \\
\hline & Number subgroups $\simeq \mathbb{Z}_{6} \times \mathbb{Z}_{18}$ & 1 \\
\hline
\end{tabular}




\section{LÁSZLÓ TÓTH}

Acknowledgements. The author gratefully acknowledges support from the Austrian Science Fund (FWF) under the project Nr. M1376-N18. The author thanks D. D. Anderson and J. Petrillo for sending him copies of their papers quoted in the References. The author is thankful to the referee for careful reading of the manuscript and for many helpful suggestions on the presentation of this paper.

\section{REFERENCES}

[1] ANDERSON, D. D.-CAMILLO, V.: Subgroups of direct products of groups, ideals and subrings of direct products of rings, and Goursat's lemma, in: Rings, Modules and Representations. Internat. Conf. on Rings and Things, Zanesville, OH, USA, 2007, Contemp. Math., Vol. 480, Amer. Math. Soc., Providence, RI, 2009, pp. 1-12.

[2] BAUER, K.-SEN, D.-ZVENGROWSKI, P.: A generalized Goursat lemma, Preprint, 2011, arXiv: 11009.0024 [math.GR].

[3] CALHOUN, W. C.: Counting the subgroups of some finite groups, Amer. Math. Monthly 94 (1987), 54-59.

[4] CĂLUGĂREANU, G.: The total number of subgroups of a finite abelian group, Sci. Math. Jpn. 60 (2004), 157-167.

[5] CRAWFORD, R. R.-WALLACE, K. D.: On the number of subgroups of index two-An application of Goursat's theorem for groups, Math. Mag. 48 (1975), 172-174.

[6] GOURSAT, É.: Sur les substitutions orthogonales et les divisions régulières de l'espace, Ann. Sci. Ècole Norm. Sup. (3) 6 (1889), 9-102.

[7] HAMPEJS, M.-HOLighaUS, N.-TÓTH, L.-WIESMEYR, C.: Representing and counting the subgroups of the group $\mathbb{Z}_{m} \times \mathbb{Z}_{n}$, Journal of Numbers, Vol. 2014, Article ID 491428.

[8] HAMPEJS, M.-TÓTH, L.: On the subgroups of finite abelian groups of rank three, Ann. Univ. Sci. Budapest. Eötvös Sect. Comput. 39 (2013), 111-124.

[9] LAMBEK, J.: Goursat's theorem and the Zassenhaus lemma, Canad. J. Math. 10 (1958), $45-56$.

[10] MACHÌ, A.: Groups. An Introduction to Ideas and Methods of the Theory of Groups. Springer, Berlin, 2012.

[11] NOWAK, W. G.-TÓTH, L.: On the average number of subgroups of the group $\mathbb{Z}_{m} \times \mathbb{Z}_{n}$, Int. J. Number Theory 10 (2014), 363-374.

[12] PETRILlO, J.: Goursat's other theorem, College Math. J. 40 (2009), 119-124.

[13] PETRILLO, J.: Counting subgroups in a direct product of finite cyclic groups, College Math. J. 42 (2011), 215-222.

[14] ROTMAN, J. J.: An Introduction to the Theory of Groups (4th ed.), in: Grad. Texts in Math., Vol. 148, Springer-Verlag, New York, 1995.

[15] TĂRNĂUCEANU, M.: An arithmetic method of counting the subgroups of a finite Abelian group, Bull. Math. Soc. Sci. Math. Roumanie (N.S.) 53(101) (2010), 373-386. 


\section{SUBGROUPS OF FINITE ABELIAN GROUPS HAVING RANK TWO}

[16] TÓTH, L.: Menon's identity and arithmetical sums representing functions of several variables, Rend. Sem. Mat. Univ. Politec. Torino 69 (2011), 97-110.

[17] TÓTH, L.: On the number of cyclic subgroups of a finite Abelian group, Bull. Math. Soc. Sci. Math. Roumanie (N.S.) 55(103) (2012), 423-428.

[18] TÓTH, L.: Multiplicative arithmetic functions of several variables: a survey, in: Mathematics Without Boundaries, Surveys in Pure Mathematics (Th. M. Rassias, P. Pardalos, eds.), Springer, New York, 2014, pp. 483-514.

Received September 29, 2014

Institute of Mathematics Universität für Bodenkultur Gregor Mendel-Straße 33

A-1180 Vienna

AUSTRIA

Department of Mathematics University of Pécs

Ifjúság u. 6

H-7624 Pécs

HUNGARY

E-mail: ltoth@gamma.ttk.pte.hu 